\title{
SONOGRAPHIC EVALUATION OF ACUTE PELVIC PAIN
}

Kamlesh Gupta ${ }^{1}$, Neelam Gauba ${ }^{2}$, Arvinder Singh ${ }^{3}$, Ramesh Chander ${ }^{4}$, Vikas Kumar ${ }^{5}$

${ }^{1}$ Professor, Department of Radiodiagnosis, SGRDIMSAR, Amritsar.

2 Professor, Department of Radiodiagnosis, GMC, Amritsar.

${ }_{3}^{3}$ Associate Professor, Department of Radiodiagnosis, GMC, Amritsar.

${ }_{4}^{4}$ Associate Professor, Department of Radiodiagnosis, GMC, Amritsar.

5Junior Resident, Department of Radiodiagnosis, GMC, Amritsar.

\section{ABSTRACT}

\section{BACKGROUND}

Acute pelvic pain can result from the gastrointestinal, gynaecological and/or urological systems. Ultrasound can be used as an initial imaging modality in the evaluation of acute pelvic pain.

\section{OBJECTIVE}

To evaluate the use of ultrasonography in acute pelvic pain, characterise the most common aetiologies and study the age and sex incidence in patients of acute pelvic pain.

\section{MATERIALS AND METHODS}

The study was conducted on hundred patients in the Department of Radiodiagnosis and Imaging, Government Medical College, Amritsar, from August 2013 to October 2015. Patients presenting with acute pelvic pain from various emergency, outpatient and indoor departments were included in the study. Based on sonographic findings provisional diagnosis was made, which was confirmed by operative findings/therapeutic response/FNAC findings/Histopathological/Laboratory findings. All pregnant patients and trauma cases were excluded from the study.

\section{RESULTS}

The age incidence of the cases in this study varied between 4 years to 56 years. The mean age of the patients in the study was 31 years; $23 \%$ cases were males and $77 \%$ cases were females in this study with the male-to-female ratio of $1: 3.34$. The appendix is most commonly involved in acute pelvic pain and is seen in 38\% cases followed by Adnexal lesions in $36 \%$ cases, urinary system in $15 \%$ cases, small bowel in $8 \%$ cases, lymph nodes in 3\% of cases. In Adnexal pathologies of acute pelvic pain, haemorrhagic ovarian cysts are most common, constituting 50\% of Adnexal lesions in acute pelvic pain followed by PID in $41.7 \%$ and ovarian torsion in $8.3 \%$ of Adnexal lesions; $15 \%$ of pathologies related to urinary system, $8 \%$ with small bowel obstruction and $3 \%$ patients with mesenteric adenitis.

\section{CONCLUSIONS}

Ultrasound is a very good modality for acute pelvic pain, as it can easily diagnose and characterise the causes of pelvic pain. The wide availability, radiation free and cost effectiveness makes it a first line investigation in acute pelvic pain.

\section{KEYWORDS}

Sonography, Acute Pelvic Pain, Radiology.

HOW TO CITE THIS ARTICLE: Gupta K, Gauba N, Singh A, et al. Sonographic evaluation of acute pelvic pain. J. Evolution Med. Dent. Sci. 2016;5(46):2914-2921, DOI: 10.14260/jemds/2016/679

\section{INTRODUCTION}

Pelvic pain is abdominal pain located below the level of the umbilicus including frequently lower back pain with or without radiation into the thighs.1 Acute pelvic pain generally implies pain that is of less than 3-month duration in a toxic, ill-appearing and unstable patient or chronic pain that is worsening. When a female in the reproductive age presents with acute pelvic and/or lower abdominal pain, the first diagnoses to consider are those that are life-threatening and would require urgent and/or emergent surgical intervention.

Financial or Other, Competing Interest: None.

Submission 15-03-2016, Peer Review 14-04-2016,

Acceptance 23-04-2016, Published 09-06-2016.

Corresponding Author:

Dr. Arvinder Singh,

Moon Ave,

Amrithsar.

E-mail: arvinderdr@rediffmail.com

DOI: $10.14260 /$ jemds/2016/679
Because the differential diagnosis of acute pelvic pain in the non-pregnant female of reproductive age includes many different organ systems (i.e. gastrointestinal, gynaecological, urological, vascular, etc.) a cost-effective and efficient strategy, such as ultrasound needs to be employed. ${ }^{2}$

Ultrasound is the technique of choice for investigation of pelvic pain, as it affords reliable diagnosis of a range of gynaecologic conditions. This is because it is readily available, non-invasive, portability, non-ionizing radiation is used and relatively low price compared with other cross-sectional imaging techniques like computed tomography and magnetic resonance imaging. ${ }^{3}$

The role of pelvic sonography in the evaluation of acute pelvic pain has been well described. Due to its higher resolution of anatomic detail, TVS should be used whenever possible, although TAS is recommended when uterine and adnexal structures are beyond the field of view of the transvaginal probe. 
In addition, duplex and colour or power Doppler imaging can be used to characterize vascularity of the ovaries, adnexal structures and uterus, information that may be helpful in narrowing the field of different considerations. ${ }^{4}$

\section{MATERIALS AND METHODS}

The present study was conducted on hundred patients in the Department of Radiodiagnosis and Imaging, Government Medical College, Amritsar from August 2012 to October 2014 after Institute Ethics Committee approval and written informed consent. Patients presenting with acute pelvic pain from various emergency, outpatient and indoor departments were included in the study.

Patients with unstable vital signs, shock, trauma and pregnant patients were specifically excluded from the study. History, clinical examination and laboratory findings were recorded. Plain X-ray of the abdomen in supine and erect position and special investigations like intravenous urography, cystography and contrast studies of the gastrointestinal tract were carried out whenever necessary.

The patient was made to lie supine on the examination table. The ultrasound coupling gel was applied to the abdomen, so as to remove air between the abdominal skin and the transducer. Patients were subjected to sonographic examination on Philips Envisor $\mathrm{C}$ ultrasound machine/Toshiba ultrasound machine modelSSA.510A/Esaote ultrasound machines using a curvilinear probe of 3.5 MHz-5 MHz or linear high frequency probe 7-12 MHz. Longitudinal, transverse and oblique views were taken. All the areas of the abdomen were examined by real time sonography including right upper quadrant, left upper quadrant, both the kidneys, lesser sac and pelvis, irrespective of the clinical diagnosis, but paying special attention to the organ involved. Based on sonographic findings, provisional diagnosis will be made. The final diagnosis was made on the basis of operative findings/therapeutic response/FNAC findings/Histopathological/Laboratory findings.

\section{RESULTS}

Hundred patients participated in this study. In the present study, the maximum no. of cases was in the age group of 31 to 40 years $(36 \%)$. The age range was between 4 to 56 years with the mean age being 31 years. There were 23 males and 77 females in this study with the male-to-female ratio of 1:3.34. Most commonly associated symptom of acute pelvic pain was nausea and vomiting in $45 \%$ of cases followed by fever in $39 \%$ cases. Other symptoms were dysuria, vaginal discharge, constipation, haematuria, and distention. Tenderness was the most common sign of acute pelvic pain seen in $50 \%$ of cases followed by abdominal tenderness, palpable pelvic mass and guarding.

The most common cause of acute pelvic pain was appendicitis in 38\% cases, adnexal lesions in 36\% cases followed by urinary system, small bowel, lymph nodes.

There were a total 38 cases of acute appendicitis. Out of these, the inflamed appendix was visualised as a noncompressible, aperistaltic tubular structure blind ended with an average diameter of more than $6 \mathrm{~mm}$ in 32 cases. The fluid filled lumen was seen in 15 cases of appendicitis with periappendicular collection in 7 cases. Appendicular lump formation presented as heterogeneous mass in right iliac fossa in 5 cases, appendicolith was seen in 4 cases. The localised enlarged lymph nodes were seen in 5 cases.
A total of 36 cases adnexal pathologies constitute acute pelvic pain cases. Haemorrhagic ovarian cysts were most common in Adnexal pathologies constituting acute pelvic pain. These constituted 18 cases, i.e. 50\% of Adnexal lesions in acute pelvic pain. PID constitutes 15 cases, i.e. $41.7 \%$ of cases. The least common adnexal pathology causing acute pelvic pain was ovarian torsion making up only $3(8.3 \%)$ cases.

Out of 36 cases of adnexal pathologies, there were 21 ovarian masses and 15 fallopian tube pathologies. The 12 of ovarian masses were on right side and 9 were on left side; of them showed size between $3-5 \mathrm{~cm}$ and 3 were having size $>5$ $\mathrm{cm}$. The cystic appearance with posterior acoustic enhancement was seen in 17 cases and heterogeneous appearance in 4 cases. The fluid debris levels were seen in 4 cases of ovarian masses. Enlarged ovaries with multiple follicles were seen in 3 cases. The thickened walls of fallopian tubes were seen in 12 cases of fallopian tube pathologies with dilated tubes in 11 cases, incomplete septa in 8 cases and 4 cases were having tubo-ovarian complexes.

A total of 15 patients presented with urinary system pathologies. Dilated pelvicalyceal system and dilated ureter were seen in 10 patients. Ureteric calculus was seen in 8 patients; 6 patients showed associated renal calculi; 5 patients presented with burning micturition. All of them had thickened bladder wall and internal echoes; 8 patients were there of small bowel obstruction presenting with complaint of acute pelvic pain. In all the patients, dilated gut loops were seen; 7 out of 8 showed increased peristalsis and interloop fluid was seen only in 5 cases out of 8 cases; 3 patients of the mesenteric adenitis presented with acute pelvic pain. All of them showed multiple enlarged lymph nodes in right inguinal fossa with size more than $10 \mathrm{~mm}$ in short axis. Thickened wall of the terminal ileum is seen in 2 cases. Localised tenderness was seen. Some of them had fever.

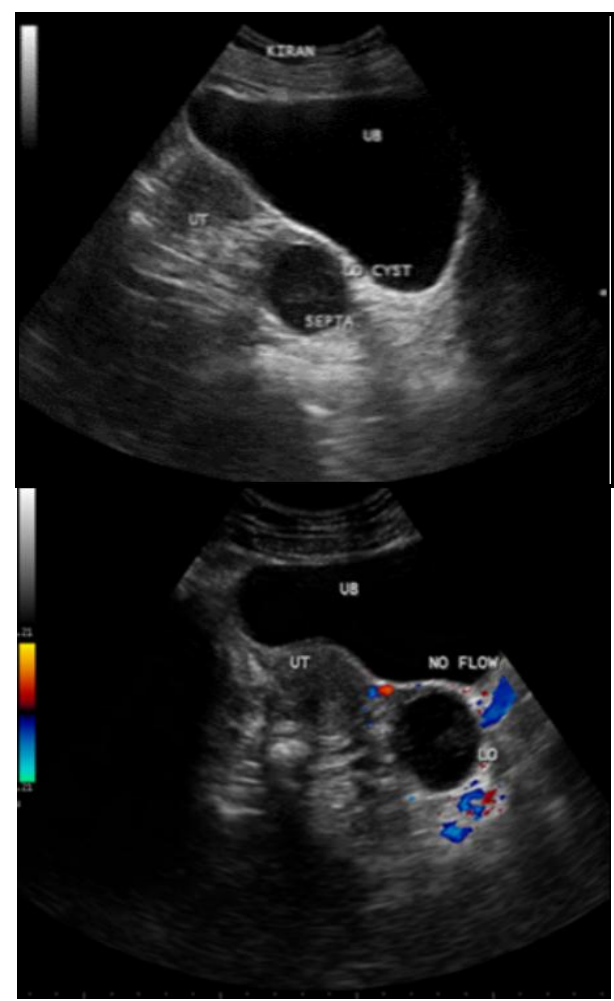

Fig. 1: Ultrasound Pelvis showing a Well-Defined Cystic Lesion with Internal Echoes and Posterior Acoustic Enhancement in Left Adnexa s/o Haemorrhagic Ovarian Cyst 


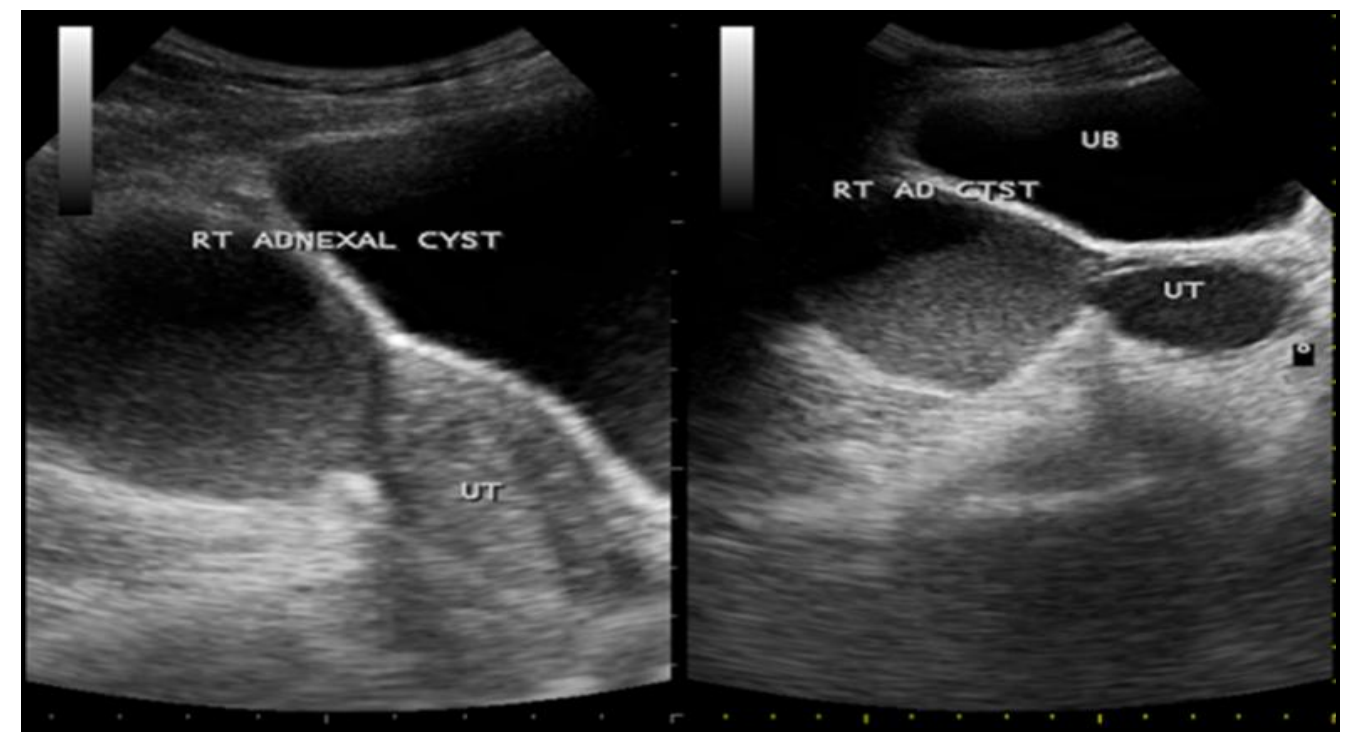

Fig. 2: Ultrasound Abdomen showing a Cystic Lesion in the Right Adnexa with Fluid Debris Level in it s/o Haemorrhagic Ovarian Cyst

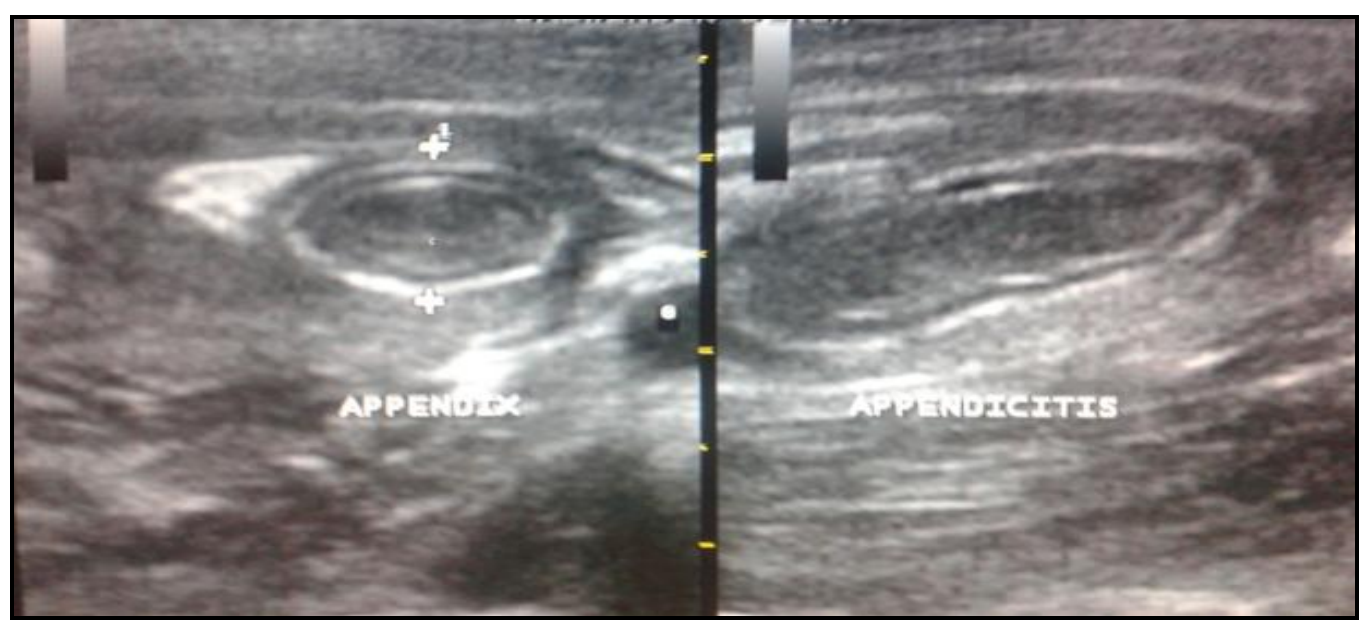

Fig. 3: Inflamed Appendix is seen as a Non-Compressible, Aperistaltic, Tubular Structure of Diameter Greater than $6 \mathrm{~mm}$ with a Blind End Arising from the Caecum in the Right Lower Quadrant. The alternating Hypo and Hyperechogenic Concentric Layers Together Constitute the Gut Signature (Calipers)

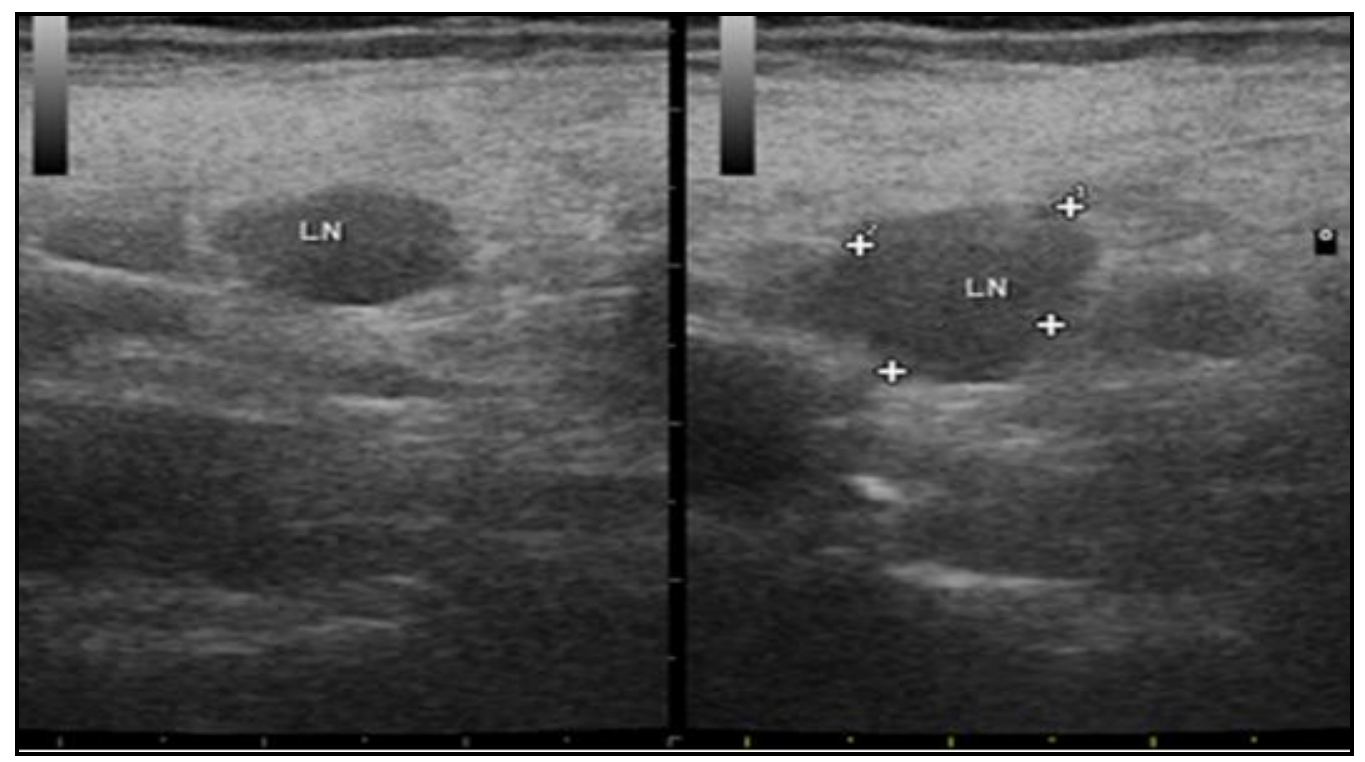

Fig. 4: Enlarged Multiple Mesenteric Nodes with Short Axis Diameter $>10 \mathrm{~mm}$ s/o Mesenteric Adenitis 


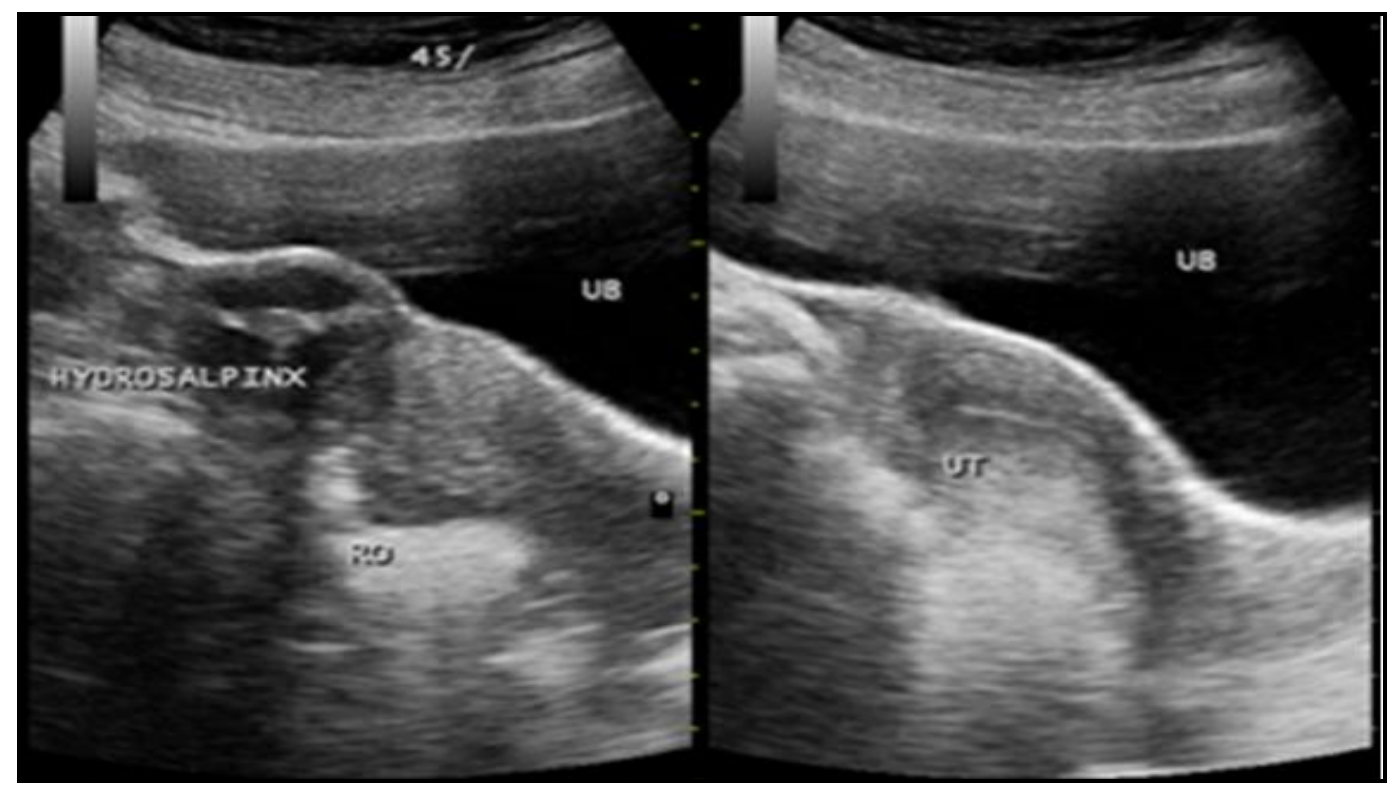

Fig. 5: Ultrasound Abdomen showing Dilated Thick Walled Fluid Filled Right Fallopian Tube with Normal Right Ovary seen Posterior to Uterus s/o Salpingitis

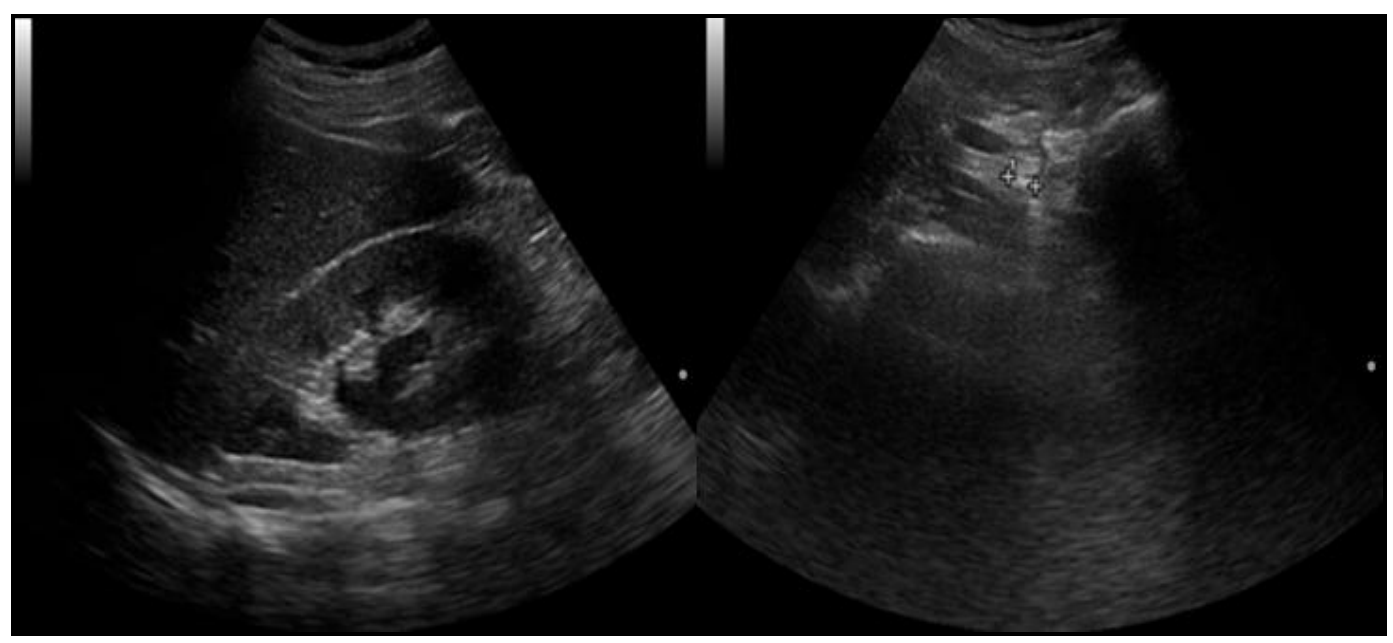

Fig. 6: Ultrasound Abdomen showing Dilated Pelvicalyceal System of Right Kidney. A Calculus Measuring $6 \mathrm{~mm}$ is seen in Middle Part of Right Ureter

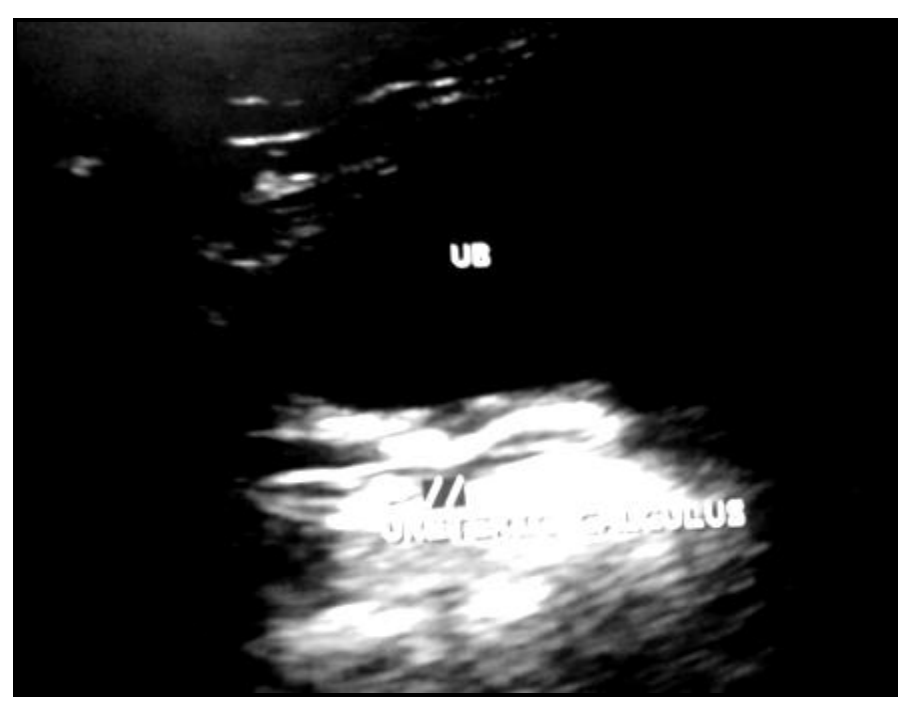

Fig. 7: Ultrasound Abdomen showing Dilated Ureter with a Calculus near its Terminal Part - Ureteric Calculus 


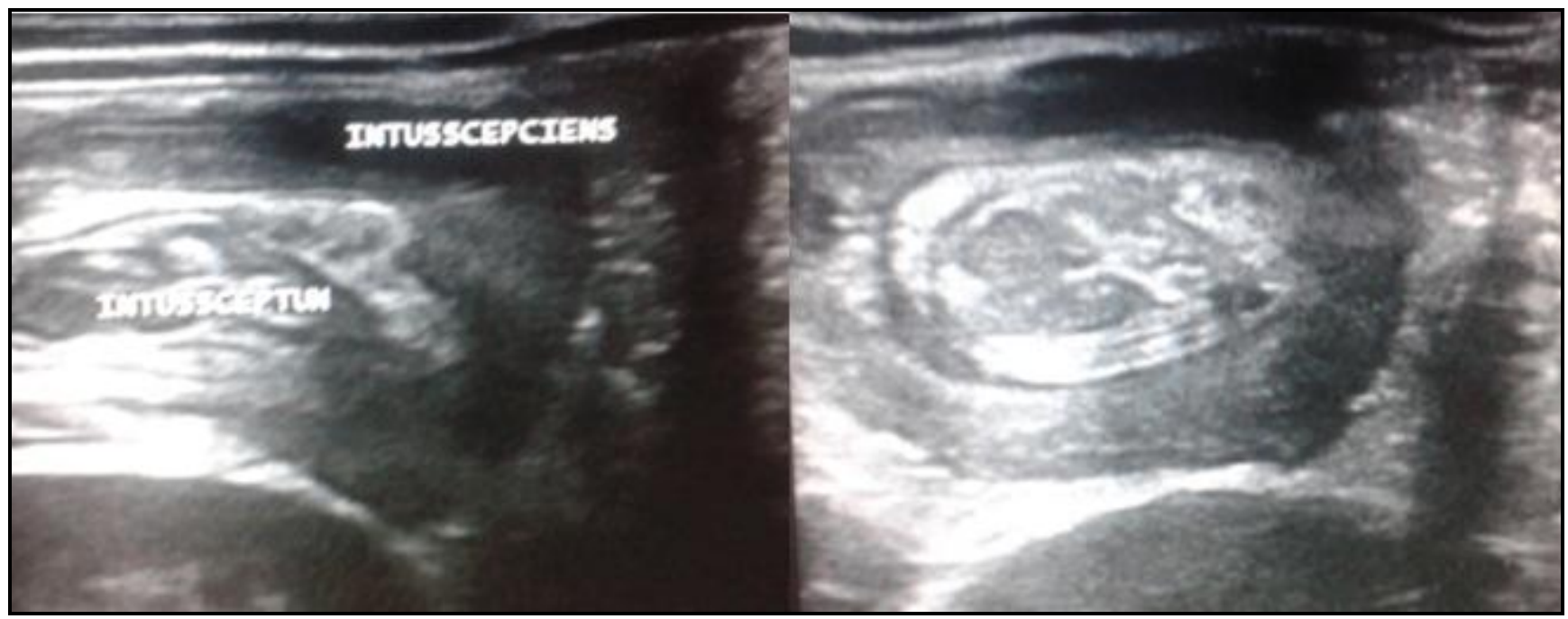

Fig. 8: Sonographic Appearance in Intussusceptions Leading to Bowel Obstruction. A Well-Defined, Round Bowel Mass with Concentric Bowel Layers and Diameter of $4.3 \mathrm{~cm}$ giving a Target/Donut Appearance is shown. The Bowel which Invaginates is known as Intussusceptum and the Bowel which receives is known as Intussuscipiens

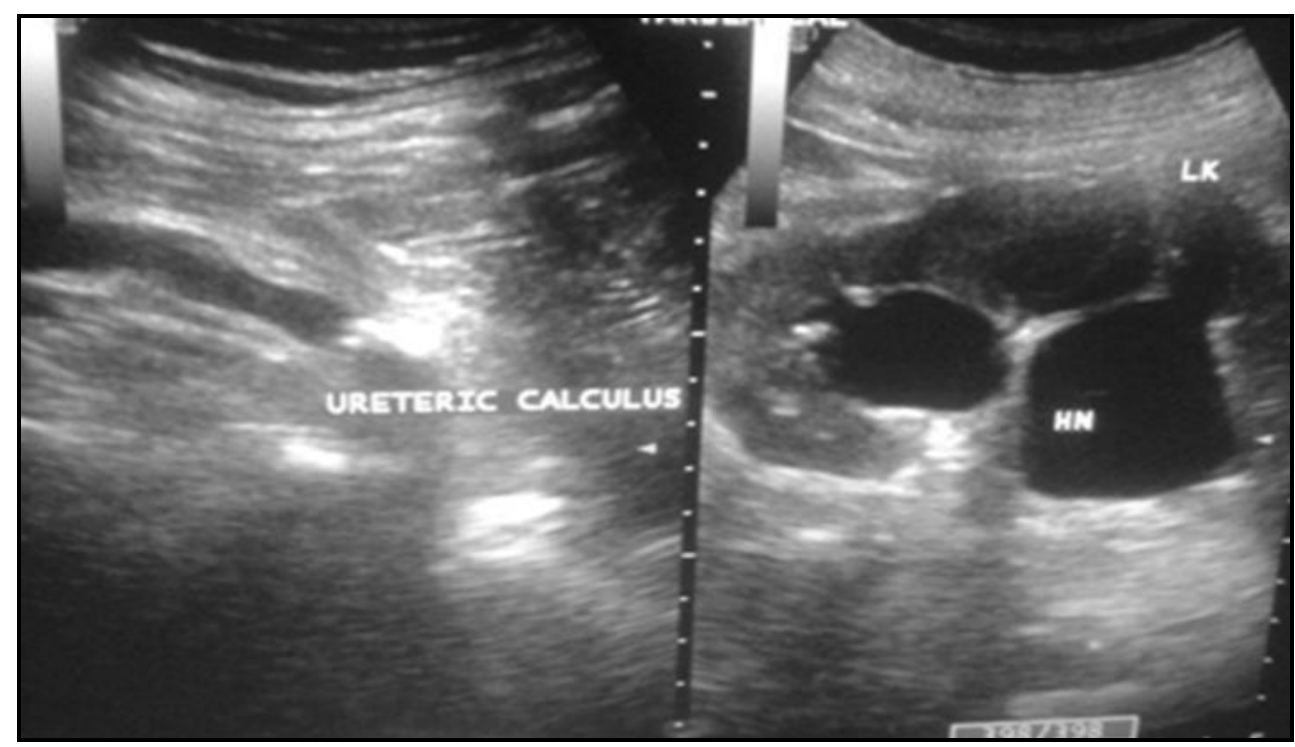

Fig. 9: Ultrasound Abdomen showing Moderate Hydronephrotic Changes with Dilated Ureter on Left Side. A Calculus with Distal Acoustic Shadowing measuring $7 \mathrm{~mm}$ is seen in the Lower One-Third of Left Ureter

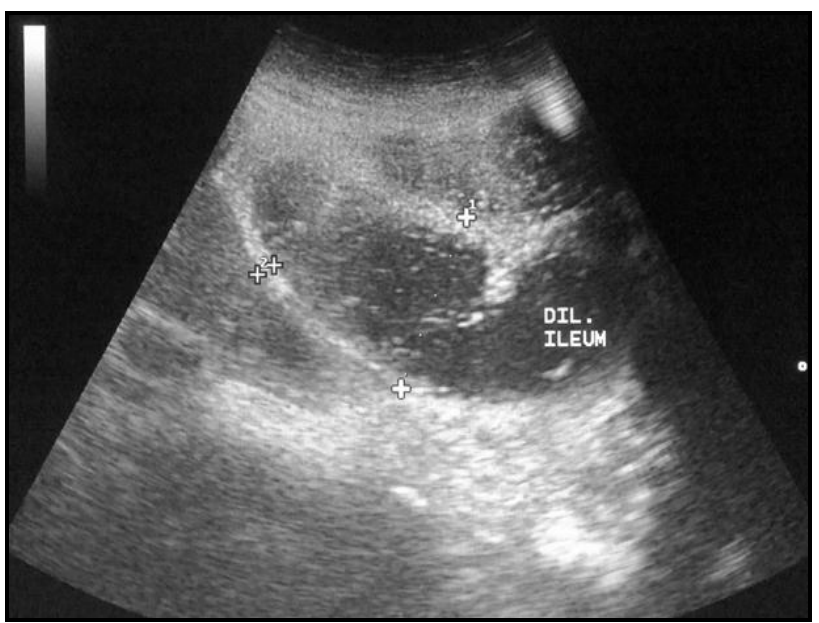

Fig. 10: Small Bowel Obstruction. Multiple Dilated, Fluid Filled, Aperistaltic Bowel Loops Are Present with a Calibre of Greater than $3.5 \mathrm{~mm}$

\section{DISCUSSION}

Acute Pelvic Pain (APP) is one of the most common complaints that gynaecologists, general surgeons and emergency service specialists encounter and for female patients to visit the emergency service. In general, APP is experienced in the lower abdomen or pelvis and lasts less than three months. ${ }^{5}$

The current study evaluated 100 patients between the age group of 4 to 56 years of age. The maximum number of cases were seen between age group of 31 to 40 , accounting up to $36 \%$ of the total cases. The mean age of cases being 31 years. This correlated well with previous study done by Sefa Kurt et al, showing the mean age of $29.9 \pm 6.01$ years. ${ }^{5}$ It also corresponds to the results of study done by Luntsi Geofry et al, in which the most common age group was 30-39 years $(52.5 \%)$ and $20-29$ years $(31.5 \%)$. The mean age of the patients was 31.6 years with standard deviation of 2.5 .

In our study, there were 23 males and 77 females with the male-to-female ratio of $1: 3.34$, so predominantly the pelvic pain occurred in female patients. 
This also goes in accordance with the previous study by W. Newton Long saying men rarely experience pelvic discomfort, whereas women frequently do. ${ }^{1}$

In present study, the most common associated symptom with acute pelvic pain is nausea and vomiting and is seen in about $45 \%$ of cases. The next common symptom is fever, dysuria, vaginal discharge, constipation, haematuria, distension are also seen. In clinical signs, tenderness is the most common sign of acute pelvic pain. It is seen in $50 \%$ of cases. The rebound tenderness, palpable mass in pelvis, guarding are also seen. Nadah Zafar et al, in their study showed the associated symptoms to be nausea, vomiting, diarrhoea, fever, flank pain, dysuria, haematuria, frequency, urgency, vaginal bleeding and vaginal discharge. ${ }^{2}$ Rochelle F. Andreotti et al shown that the clinical diagnosis of acute pelvic pain in the female patient can be challenging because of nonspecific signs and symptoms, and imaging has been found to be valuable in narrowing the differential diagnosis. ${ }^{6}$

In our study appendix is most commonly involved in acute pelvic pain and is seen in $38 \%$ cases. Adnexal lesions are second most common and constitute $36 \%$ cases. Then urinary system, small bowel and lymph nodes are involved in $15 \%, 8 \%, 3 \%$ of cases. In another study Appendicitis is the most common cause of right lower quadrant pain. Appendicitis is also the most common diagnosis among nongynaecological disorders that cause acute pelvic pain. ${ }^{7}$ In another study, most common non-gynaecologic APP factor causing pelvic pain was acute appendicitis. The second most common non-gynaecological APP factor was nephrolithiasis. The most common gynaecological cause for APP was ovarian cysts. ${ }^{5}$ In another study pelvic inflammatory disease is one of the most common causes of acute pelvic pain in women. ${ }^{8}$

In our study there were total 38 confirmed cases of acute appendicitis, out of which 32 were correctly diagnosed with ultrasound with a sensitivity of $84 \%$. It correlates with the study of Bernard A. Birnbaum who reported sensitivities of $75 \%-90 \%$, specificities of $86 \%-100 \%$ for the diagnosis of acute appendicitis. ${ }^{9}$ Out of these, the inflamed appendix was visualised as a non-compressible, aperistaltic tubular structure blind ended with an average diameter of more than $6 \mathrm{~mm}$ in 32 cases, i.e. 84.2\%. It correlates well with the study of Jerry L. Old showing that the most useful finding on ultrasonography that is suggestive of appendicitis is an outer appendiceal diameter of $6 \mathrm{~mm}$ or greater on cross section. ${ }^{10}$ In 7 cases $(18 \%)$ diagnosed on ultrasonography, there was localised collection in right iliac fossa. Carlos J. Sivit showed in study that localised periappendiceal collections are seen in the right lower quadrant or pelvis. ${ }^{11}$ Appendicolith was seen in 4 cases $(10.5 \%)$.

It correlated well with Emil J. Balthazar et al study on patients with acute appendicitis revealing appendicoliths in $17 \%$ of cases of acute appendicitis. ${ }^{12}$ Inflamed appendix presented as heterogeneous mass in right iliac fossa in 5 cases (13\%). It was ill defined in 4 and well defined in 1 case. It correlated well with the study of Carlos J. Sivit showing periappendiceal mass in $17 \%$ of cases of acute appendicitis. ${ }^{11}$ Another study by Emil J. Balthazar et al showed paracaecal inflammatory mass and/or abscess in 7 out of 38 cases. $(18.9 \%){ }^{12}$ Fluid filled lumen was seen in 15 cases of appendicitis amounting to $39.47 \%$. This correlated well with the study of Carlos J. Sivit, in which $47 \%$ cases show fluid filled lumen. ${ }^{11}$ Enlarged lymph nodes were seen in 5 cases
(13.1\%) in our study. Nicolas Kessler et al had shown that right lower abdominal quadrant adenopathy is a common reaction to ileal, caecal or appendiceal inflammatory disease that is encountered in both patients with appendicitis and those without it. 13

In our study, total 36 cases of adnexal pathologies constitute acute pelvic pain cases. Haemorrhagic ovarian cysts are most common in Adnexal pathologies constituting acute pelvic pain. These constitute 18 cases, i.e. $50 \%$ of Adnexal lesions in acute pelvic pain. It correlated well with the study of Kaisuke Ishihara et al, which showed that Haemorrhagic Ovarian Cyst (HOC) is often involved in acute abdomen. ${ }^{14}$ PID constitute 15 cases, i.e. $41.7 \%$ of cases. This correlated well with a previous study, in which salpingitis and haemorrhagic ovarian cysts are most commonly diagnosed gynaecologic conditions presenting as an acute abdomen.15 Least common Adnexal pathology constituting acute pelvic pain in this study is ovarian torsion making up only $8.3 \%$ of cases (3 cases). It also correlated well with the study of Sandra 0. Allison et al that Gynaecologic disorders in the woman with a negative pregnancy test who presents with acute pelvic pain include acute Pelvic Inflammatory Disease (PID), functional ovarian cysts, ovarian endometriomas and adnexal torsion. ${ }^{16}$

In our study, there were 21 ovarian pathologies and 15 fallopian tube pathologies in Adnexal lesions. The 12 (57.1\%) of ovarian masses were on right side and $9(42.9 \%)$ were on left side. Leena Mawaldi et al stated that the right ovary is more likely than the left to undergo torsion. ${ }^{17} ; 18$ of ovarian masses $(85.7 \%)$ showed size to be between 3 to $5 \mathrm{~cm}$ and 3 $(14.3 \%)$ were having size more than $5 \mathrm{~cm}$. Kiran A. Jain stated that the average diameter of the haemorrhagic ovarian cyst is 3.0 to $3.5 \mathrm{~cm} .{ }^{18}$ Debra Houry et al stated that the mean size of the torsed ovary at surgery was $9.5 \mathrm{~cm}$ (Range 1 to $30 \mathrm{~cm}$ ); $77(89 \%)$ patients had an ovary greater than $5 \mathrm{~cm} .{ }^{19}$ Cystic appearance with posterior acoustic enhancement were seen in $17(81 \%)$ of ovarian masses and $4(11.1 \%)$ were having heterogeneous appearance.

This also goes in accordance with Kiran A. Jain study stating that posterior enhanced through transmission is seen signifying the basic cystic nature of the haemorrhagic ovarian cyst. 18 Oksana H. Baltarowich stated that most haemorrhagic ovarian cysts appear as masses with heterogeneous echogenicity with the largest subgroup being predominantly anechoic masses containing hypoechoic material. ${ }^{20}$ Leena Mawaldi study stated the Gray-scale findings of ovarian torsion typically including asymmetric enlargement, a solid heterogeneous appearance and peripheral cystic areas. ${ }^{17}$ Reduction in size was seen in 16 (76\%) of ovarian masses on followup study. Maitray D. Patel et al stated that short interval follow-up sonography of haemorrhagic ovarian cyst cases will nearly always show resolution of the mass. ${ }^{21}$ Fluid debris levels were seen in 4 (19\%) cases of ovarian masses. Kiran A. Jain stated that a Haemorrhagic ovarian cyst can have a fluid-fluid or fluid-debris level. ${ }^{8}$ Enlarged ovaries with multiple follicles are seen in 3 cases $(14.2 \%)$ of ovarian masses. M. Graif et al stated in their study that sonographic features of diffuse swelling of the ovarian parenchyma and follicular enlargement in the cortical zone were highly suggestive for torsion of the ovary. ${ }^{22}$ Thick walls of fallopian tubes were seen in 12 cases (80\%) of fallopian tube pathologies. I. E. Timor-Tritsch et al in their study stated that 
$100 \%$ cases of acute tubal inflammatory disease have thick walls of fallopian tubes. ${ }^{23}$

Dilated tubes were seen in 11 cases (73.35\%) of fallopian tube pathologies. This also correlated well with the study of Jose Bajo Arenas et al, which states that dilated fluid filled tube is the most frequent result of pelvic inflammatory disease. ${ }^{24}$ Incomplete septa were seen in 8 cases (53.3\%) of the fallopian tube pathologies. This correlated with the I. E. Timor-Tritsch et al study, which states that incomplete septa are seen in $86 \%$ of acute pelvic infection. ${ }^{23}$ This also correlated with the study of Jose Bajo Arenas et al, which states that $35.25 \%$ of cases of acute pelvic inflammatory disease have incomplete septa. ${ }^{24}$ In our study, 4 cases $(26.7 \%)$ were having tubo-ovarian complexes. This also correlated with the study of Jose Bajo Arenas et al, which states that $23.4 \%$ of acute pelvic inflammatory disease patients have tubo-ovarian complexes. ${ }^{24}$ Free fluid in POD was seen in 16 cases (44.4\%) of all the Adnexal pathologies. This correlates well with the I. E. Timor-Tritsch et al study, which states that cul-de-sac fluid is more commonly seen in the acute cases. Seven out of the $14(50 \%)$ showed some fluid in the cul-de-sac in their study. ${ }^{23}$

In our study, total 15 patients presented with acute pelvic pain with pathologies related to urinary system. Dilated pelvicalyceal system and dilated ureter were seen in 10 patients $(100 \%)$ of ureteral calculus. Paul H. Ellenbogen et al in their study stated that hydronephrosis is correctly diagnosed by ultrasound in obstructed kidneys with a sensitivity of $98 \%{ }^{25}$ Ureteric calculus was seen in 8 patients with a sensitivity of $80 \%$. This correlated with the study of Seong Jin Park et al, showing the overall sensitivity of sonography for detecting ureteral calculi were $98.3 \%{ }^{26}$ Six patients showed associated renal calculi. Thickened bladder wall and internal echoes were seen in 5 cases. It correlated well with the study of Richard D. Bellah et al, which stated that irregular bladder wall thickening is seen in acute cases of urinary tract infections. ${ }^{27}$

In our study, there were 8 patients of small bowel obstruction presenting with complaint of acute pelvic pain. In all the 8 patients (100\%), dilated gut loops were seen; 7 out of 8 cases $(87.5 \%)$ showed to and fro increased peristalsis. Anna Catarina Silva et al stated that at sonography, bowel obstruction is considered to be present when the lumen of the fluid filled small bowel loops is dilated to more than $3 \mathrm{~cm}$, peristalsis of the dilated segment is increased as shown by the to-and-fro or whirling motion of the bowel contents. ${ }^{28}$ Interloop fluid was seen only in 5 cases out of 8 cases (62.5\%). This also correlated well with the Roberto Grassi et al study which states that altered peristaltic activity, thin bowel walls, fluid filled loops with hyperechoic spots in the bowel segment proximal to obstruction, presence of free extraluminal fluid is seen in intestinal obstruction on ultrasound. 29

In our study, there were 3 patients of the mesenteric adenitis presenting with acute pelvic pain. All of them $(100 \%)$ showed multiple enlarged lymph nodes in right inguinal fossa with size more than $10 \mathrm{~mm}$ in short axis. Thickened wall of the terminal ileum is seen in 2 cases $(66.7 \%)$. In a study by Natalia Simanovsky et al stated that enlarged abdominal lymph nodes of $10 \mathrm{~mm}$ or greater in their shortest axis in the clinical setting of abdominal pain may represent mesenteric lymphadenitis. 30

\section{CONCLUSION}

Acute pelvic pain is one of the most common complaints that gynaecologists, general surgeons and emergency service specialists encounter and for female patients to visit the emergency service. In present study, we conclude that ultrasound is a very good modality of choice for acute pelvic pain, as it can easily diagnose and characterise the causes of pelvic pain. The most common cause of pelvic pain is appendicitis. The wide availability, radiation free and cost effectiveness makes it a first line investigation in acute pelvic pain. The results of the present study correlated well with the previous studies. We expect present study to add existing knowledge on this topic.

\section{REFERENCES}

1. Long WN. Pelvic Pain. In: Walker HK, Hall WD, Hurst JW, eds. Clinical methods: the history, physical, and laboratory examinations. Boston: Butterworths Ch. 171, 1990;3rd edn:p 805-6.

2. Zafar N, Kupesic Plavsic S. Role of ultrasound in the evaluation of acute pelvic pain in nonpregnant reproductive age patients. Donald School J Ultrasound Obstet Gynaecol 2012;6(2):207-17.

3. Geofry L, Chigozie NI, Yusuf FA, et al. Pattern of gynaecological pelvic ultrasound findings among women with pelvic pain in a tertiary hospital in kano north western Nigeria. Journal of Dental and Medical Sciences 2015;14(7):79-82.

4. Andreotti RF, Lee SI, DeJesus Allison SO, et al. ACR appropriateness criteria: acute pelvic pain in the reproductive age group. Ultrasound Q 2011;27(3):205-10.

5. Kurt S, Uyar I, Demirtaş Ö, et al Acute pelvic pain: evaluation of 503 cases. Arch Iran Med 2013;16(7):397400.

6. Andreotti RF, Harvey SM. Sonographic evaluation of acute pelvic pain. J Ultrasound Med 2012;31(11):1713-18.

7. Bau A, Atri M. Acute female pelvic pain: ultrasound evaluation. Semin Ultrasound CT MR 2000;21(1):78-93.

8. Jain K. Gynaecologic causes of acute pelvic pain: ultrasound imaging. Ultrasound Clinics 2008;3:1-12.

9. Birnbaum BA, Wilson SR. Appendicitis at the millennium. Radiology 2000;215(2):337-48.

10. Old JL, Dusing RW, Yap W, et al. Imaging for suspected appendicitis. Am Fam Physician 2005;71(1):71-8.

11. Sivit CJ. Diagnosis of acute appendicitis in children: spectrum of sonographic findings. AJR Am J Roentgenol 1993;161(1):147-52.

12. Balthazar EJ, Birnbaum BA, Yee J, et al Acute appendicitis: CT and US correlation in 100 patients. Radiology 1994;190(1):31-5.

13. Kessler N, Cyteval C, Gallix B, et al. Appendicitis: evaluation of sensitivity, specificity, and predictive values of US, Doppler US, and laboratory findings. Radiology 2004;230(2):472-8.

14. Ishihara K, Nemoto Y. Sonographic appearance of haemorrhagic ovarian cyst with acute abdomen by transvaginal scan. J Nippon Med Sch 1997;64(5):411-5.

15. Kupesić S, Aksamija A, Vucić N, et al. Ultrasonography in acute pelvic pain. Acta Med Croatica 2002;56(4-5):171-80.

16. Allison SO, Lev-Toaff AS. Acute pelvic pain: what we have learned from the ER. Ultrasound Q 2010;26(4):211-8. 


\section{Jemds.com}

17. Mawaldi L, Gupta C, Bakhs $\mathrm{H}$, et al. Validity of ultrasound in patients with acute pelvic pain related to suspected ovarian torsion. Surgical Science 2011;2:326-30.

18. Jain KA. Sonographic spectrum of haemorrhagic ovarian cysts. J Ultrasound Med 2002;21(8):879-86.

19. Houry D, Abbott JT. Ovarian torsion: a fifteen-year review. Ann Emerg Med 2001;38(2):156-9.

20. Baltarowich $\mathrm{OH}$, Kurtz $\mathrm{AB}$, Pasto ME, et al. The spectrum of sonographic findings in haemorrhagic ovarian cysts. AJR Am J Roentgenol 1987;148(5):901-5.

21. Patel MD, Feldstein VA, Filly RA. The likelihood ratio of sonographic findings for the diagnosis of haemorrhagic ovarian cysts. J Ultrasound Med 2005;24(5):607-14.

22. Graif M, Itzchak Y. Sonographic evaluation of ovarian torsion in childhood and adolescence. AJR Am J Roentgenol 1988;150(3):647-9.

23. Timor-Tritsch IE, Lerner JP, Monteagudo A, et al. Transvaginal sonographic markers of tubal inflammatory disease. Ultrasound Obstet Gynaecol 1998;12(1):56-66.

24. Arenas JB, Perez-Medina T. Sonography of the pelvic infection. Donald School J Ultrasound Obstet Gynaecol 2012;6(4):398-407.
Original Article

25. Ellenbogen PH, Scheible W, Talner LB, et al. Sensitivity of gray-scale ultrasound in detecting urinary tract obstruction. AJR 1978;130(4):731-3.

26. Park SJ, Yi BH, Lee HK, et al. Evaluation of patients with suspected ureteral calculi using sonography as an initial diagnostic tool: how can we improve diagnostic accuracy? J Ultrasound Med 2008;27(10):1441-50.

27. Bellah RD, Epelman MS, Darge K. Sonography in the evaluation of paediatric urinary tract infection. Ultrasound Clin 2010;5:1-13.

28. Silva AC, Pimenta M, Guimarães LS. Small bowel obstruction: what to look for. Radiographics 2009;29(2):423-39.

29. Grassi R, Romano S, D'Amario F, et al. The relevance of free fluid between intestinal loops detected by sonography in the clinical assessment of small bowel obstruction in adults. Eur J Radiol 2004;50(1):5-14.

30. Simanovsky N, Hiller N. Importance of sonographic detection of enlarged abdominal lymph nodes in children. J Ultrasound Med 2007;26(5):581-4. 